\title{
STABILIZED CROUZEIX-RAVIART ELEMENT FOR THE DARCY-STOKES PROBLEM
}

\author{
ERIK BURMAN* AND PETER HANSBO ${ }^{\dagger}$
}

\begin{abstract}
We stabilize the nonconforming Crouzeix-Raviart element for the Darcy-Stokes problem with terms motivated by a discontinuous Galerkin approach. Convergence of the method is shown, also the in limit of vanishing viscosity. Finally, some numerical examples verifying the theoretical predictions are presented.
\end{abstract}

AMS subject classifications. $65 \mathrm{~N} 12,65 \mathrm{~N} 30,76 \mathrm{D} 07$

Key words. Stokes problem, Darcy's problem, generalized Stokes problem, Crouzeix-Raviart element

1. Introduction. The Darcy-Stokes problem is interesting for a variety of reasons. Apart from being a modeling tool in its own right, it also appears, less obviously, in time-stepping methods for Stokes and for high Reynolds number flows (where of course the convective term causes addidtional difficulties). It would thus be advantageous if the same element could be used in both the Stokes limit and the Darcy limit. One obvious candidate for such an element is the nonconforming Crouzeix-Raviart (CR) element, which has several nice properties: in combination with piecewise constant pressures it satisfies the inf-sup condition and is elementwise mass conserving; it is also easy to implement. However, in a recent paper by Mardal, Tai, and Winther [8] it is shown that the CR element does not converge when applied to the Darcy problem (or the Darcy-Stokes problem with vanishing viscosity). It is also well known that the CR element does not fulfill a discrete Korn's inequality which precludes the use of the physically more realistic form of the Stokes operator. Stabilization sufficient for fulfilling the Korn's inequality was introduced by Hansbo and Larson [7] for the elasticity operator. In this paper we also show that a similar stabilization for a mixed finite element method, with piecewise constant pressure, for the Darcy-Stokes problem will ensure convergence in the Darcy limit.

For the Darcy problem, it turns out that our method yields a better convergence numerically, $O\left(h^{2}\right)$, for the velocities than is to be expected from our analysis, which gives errors of $O(h)$ (here $h$ is the mesh size parameter). The reason we cannot obtain second order convergence in the analysis is that the error in the divergence term appears in the form of the nonconformity in the normal jump emanating from the Crouzeix-Raviart approximation. Thus the error in divergence is still present which allows pollution from the poor approximation of the pressure. In $H$ (div) conforming methods there is no such term, and the problem is instead to obtain the full polynomial approximation necessary for the analysis in the Stokes case. Examples of elements with normal continuity and full polynomial approximation include the BDM element [2], which however is nonconforming in the Stokes case, and the construction in [8]. We remark that a drawback of such approximations is that they are geometry-dependent and cannot be constructed on a reference element, unlike the CR element.

\footnotetext{
*Department of Mathematics, Ecole Polytechnique Federale de Lausanne, Switzerland

†Department of Applied Mechanics, Chalmers University of Technology, SE-41296 Göteborg, Sweden
} 
2. Problem statement. In this paper we will consider a Darcy-Stokes problem of the following type

$$
\begin{aligned}
& \sigma \boldsymbol{u}-2 \mu \nabla \cdot \varepsilon(\boldsymbol{u})+\nabla p=f \quad \text { in } \Omega, \\
& \nabla \cdot \boldsymbol{u}=g \text { in } \Omega
\end{aligned}
$$

Here $\Omega$ is an open subset of $\mathbb{R}^{d}, d=2$ or $d=3$, with outward pointing normal $\boldsymbol{n}, \boldsymbol{u}$ is the velocity vector, $p$ is the pressure, $\varepsilon(\boldsymbol{u})=\left[\varepsilon_{i j}(\boldsymbol{u})\right]_{i, j=1}^{d}$ is the symmetric part of the velocity gradient tensor with components

$$
\varepsilon_{i j}(\boldsymbol{u})=\frac{1}{2}\left(\frac{\partial u_{i}}{\partial x_{j}}+\frac{\partial u_{j}}{\partial x_{i}}\right)
$$

$\nabla \cdot \varepsilon=\left[\sum_{j=1}^{d} \partial \varepsilon_{i j} / \partial x_{j}\right]_{i=1}^{d}, \boldsymbol{I}=\left[\delta_{i j}\right]_{i, j=1}^{2}$ with $\delta_{i j}=1$ if $i=j$ and $\delta_{i j}=0$ if $i \neq j$, $\operatorname{tr} \varepsilon(\boldsymbol{u})=\sum_{k} \varepsilon_{k k}(\boldsymbol{u})=\nabla \cdot \boldsymbol{u}$, and $\boldsymbol{f}$ and $g$ are given force terms. Furthermore, $\mu \geq 0$ is the viscosity constant and $\sigma \geq 0$ is a permeability constant. We assume that either $\sigma \geq 1$ or $\mu \geq 1$. For simplicity we assume Dirichlet conditions on the boundary, that is, $\boldsymbol{u}=0$ on $\partial \Omega$ for Stokes and $\boldsymbol{u} \cdot \boldsymbol{n}=0$ on $\partial \Omega$ in the limit case $\mu=0$, i.e., Darcy flow.

The main difference between Stokes and Darcy's equations, from the point of view of analysis, is that in Stokes the velocities are $\left[H^{1}(\Omega)\right]^{d}$ whereas in the case of Darcy they are only in $H(\operatorname{div} ; \Omega)$. This loss of regularity must be accounted for in the analysis, and this is the main reason why the stabilized mixed nonconforming CR element combined with piecewise constant pressures is an ideal candidate for the problem: since the incompressibility condition is tested with constants the solution satisfies the incompressibility condition exactly on each element.

In this paper we apply this mixed stabilized method to Stokes' equations and Darcy's equations in a unified manner and prove optimal a priori estimates in the energy norm applying to both systems. We also give numerical examples showing the performance of the method on the separate problems.

3. Finite element formulation. In order to formulate our finite element method we first introduce the weak formulation of problem (2.1). We introduce the Hilbert spaces

$$
W^{D}=\left\{v \in H_{\operatorname{div}}(\Omega): \boldsymbol{v} \cdot \boldsymbol{n}=0 \text { on } \partial \Omega\right\},
$$

for the case $\mu=0$,

$$
W^{S}=\left\{\boldsymbol{v} \in\left[H_{0}^{1}(\Omega)\right]^{d}\right\},
$$

for $\mu>c>0$, and

$$
L_{0}^{2}=\left\{q \in L^{2}(\Omega): \int_{\Omega} q \mathrm{~d} x=0\right\},
$$

with $\Omega$ some open subset of $\mathbb{R}^{d}$. We denote the product space $W^{X} \times L_{0}^{2}$ by $\mathcal{W}^{X}$ where $X$ is chosen to $D$ or $S$ depending on the choice of equation. Let

$$
a(\boldsymbol{u}, \boldsymbol{v}):=\int_{\Omega} 2 \mu \varepsilon(\boldsymbol{u}): \varepsilon(\boldsymbol{v}) d \Omega+\int_{\Omega} \sigma \boldsymbol{u} \cdot \boldsymbol{v} d \Omega
$$


and consider the bilinear form

$$
B[(\boldsymbol{u}, p),(\boldsymbol{v}, q)]=a(\boldsymbol{u}, \boldsymbol{v})-(p, \nabla \cdot \boldsymbol{v})+(q, \nabla \cdot \boldsymbol{u}) .
$$

The weak formulation of (2.1) now takes the form, find $(u, p) \in \mathcal{W}^{X}$ such that

$$
B[(\boldsymbol{u}, p),(\boldsymbol{v}, q)]=(f, \boldsymbol{v}) \quad \forall(\boldsymbol{v}, q) \in \mathcal{W}^{X}
$$

Let $\mathcal{T}_{h}$ be a conforming, shape regular triangulation of $\Omega$ and $\mathcal{E}_{h}$ denote the set of all element sides in the mesh. We introduce the non-conforming Crouzeix-Raviart finite element space of piecewise linears and piecewise constants

$$
V_{h}:=\left\{\boldsymbol{v}:\left.\boldsymbol{v}\right|_{K} \in\left[P_{1}(K)\right]^{d}: \int_{e}[\boldsymbol{v}] d s=0, \forall e \in \mathcal{E}_{h}\right\},
$$

where $[\boldsymbol{v}]$ denotes the jump across the edge for internal edges and $[\boldsymbol{v}]=\boldsymbol{v}$ for $e \cap \partial \Omega \neq$ $\emptyset$. Further,

$$
Q_{h}:=\left\{q:\left.q\right|_{K} \in P_{0}(K): \int_{\Omega} q \mathrm{~d} x=0\right\}
$$

We will also use the space

$$
W_{h}:=\left\{\boldsymbol{v} \in V_{h}: \sum_{K} \int_{K} \nabla \cdot \boldsymbol{v} q d x=0 \quad \forall q \in Q_{h}\right\} .
$$

It is well known (see, e.g., [2]) that the spaces $V_{h}, Q_{h}$ satisfy the inf-sup condition and hence $W_{h}$ is non-empty.

We introduce the following bilinear form on which we will base our finite element method

$$
\begin{aligned}
B_{h}[(\boldsymbol{u}, p),(\boldsymbol{v}, q)]= & a_{h}(\boldsymbol{u}, \boldsymbol{v})-(p, \nabla \cdot \boldsymbol{v})_{h} \\
& +(q, \nabla \cdot \boldsymbol{u})_{h}+J_{\mu}(\boldsymbol{u}, \boldsymbol{v})+J_{0}(\boldsymbol{u}, \boldsymbol{v})
\end{aligned}
$$

where

$$
\begin{gathered}
a_{h}(\boldsymbol{u}, \boldsymbol{v})=\int_{\Omega} \sigma \boldsymbol{u} \cdot \boldsymbol{v} d \Omega+\sum_{K} \int_{K} 2 \mu \varepsilon(\boldsymbol{u}): \varepsilon(\boldsymbol{v}) d x \\
(p, \nabla \cdot \boldsymbol{v})_{h}=\sum_{K} \int_{K} p \nabla \cdot \boldsymbol{v} d x \\
J_{\mu}(\boldsymbol{u}, \boldsymbol{v})=\sum_{K} \gamma_{\mu} \int_{\partial K} \frac{\mu}{h_{K}}[\boldsymbol{u}] \cdot[\boldsymbol{v}] \mathrm{d} s
\end{gathered}
$$

and

$$
J_{0}(\boldsymbol{u}, \boldsymbol{v})=\sum_{K} \gamma_{0} \int_{\partial K} \frac{1}{h_{K}}[\boldsymbol{n} \cdot \boldsymbol{u}][\boldsymbol{n} \cdot \boldsymbol{v}] \mathrm{d} s,
$$

where, for definiteness, $\boldsymbol{n}$ is the outward unit normal to $K$. Below, we shall use the shorthand notation

$$
j(\boldsymbol{u}, \boldsymbol{v}):=J_{\mu}(\boldsymbol{u}, \boldsymbol{v})+J_{0}(\boldsymbol{u}, \boldsymbol{v})
$$


REMARK 3.1. The point of the jump terms is as follows. In [1] it was shown that $J_{\mu}(\boldsymbol{u}, \boldsymbol{v})$ controls the rigid body rotations which cause a lack of coercivity for the Crouzeix-Raviart approximation when applied to the Stokes problem written in terms of the symmetric part of the velocity gradient (cf. also [7]). The term $J_{0}(\boldsymbol{u}, \boldsymbol{v})$ is necessary in the Darcy limit to control the nonconformity emanating from the pressure term.

REMARK 3.2. The jump terms can be motivated by applying the discontinuous Galerkin method proposed by Hansbo and Larson [6] for the mixed form of the elasticity equations to the Crouzeix-Raviart approximation.

We propose the following finite element formulation: find $\left(\boldsymbol{u}_{h}, p_{h}\right) \in V_{\times} Q_{h}$ such that

$$
B_{h}\left[\left(\boldsymbol{u}_{h}, p_{h}\right),\left(\boldsymbol{v}_{h}, q_{h}\right)\right]=\left(f, \boldsymbol{v}_{h}\right), \quad \forall\left(\boldsymbol{v}_{h}, q_{h}\right) \in V_{h} \times Q_{h} .
$$

This finite element formulation is simply the standard Galerkin formulation with the penalizing terms $J_{\mu}\left(\boldsymbol{u}_{h}, \boldsymbol{v}_{h}\right)$ and $J_{0}\left(\boldsymbol{u}_{h}, \boldsymbol{v}_{h}\right)$ added.

Lemma 3.3. For $\boldsymbol{v} \in W_{h}$, we have $\left.\nabla \cdot \boldsymbol{v}\right|_{K}=0 \quad \forall K \in \mathcal{T}_{h}$.

Proof. Since $\nabla \cdot \boldsymbol{v}$ is a constant on each $K$ for $\boldsymbol{v} \in V_{h}$, we may take $q=\nabla \cdot \boldsymbol{v}$ on $K, q=0$ elsewhere, leading to

$$
0=\int_{K}|\nabla \cdot \boldsymbol{v}|^{2} d x=\operatorname{meas}(K)|\nabla \cdot \boldsymbol{v}|_{K}^{2}
$$

which is the statement of the lemma.

4. Stability. For the stability analysis, we consider first the following reduced problem: Find $\boldsymbol{u}_{h} \in W_{h}$ such that

$$
a_{h}\left(\boldsymbol{u}_{h}, \boldsymbol{v}_{h}\right)+j\left(\boldsymbol{u}_{h}, \boldsymbol{v}_{h}\right)=\left(\boldsymbol{f}, \boldsymbol{v}_{h}\right) \quad \forall \boldsymbol{v}_{h} \in W_{h} .
$$

On $W_{h}$ we have

$$
\begin{aligned}
a_{h}\left(\boldsymbol{u}, \boldsymbol{v}_{h}\right)+j\left(\boldsymbol{u}, \boldsymbol{v}_{h}\right)= & \left(\sigma \boldsymbol{u}, \boldsymbol{v}_{h}\right)+\left(2 \mu, \boldsymbol{\varepsilon}(\boldsymbol{u}), \boldsymbol{\varepsilon}\left(\boldsymbol{v}_{h}\right)\right)_{h} \\
= & \left(\sigma \boldsymbol{u}-\nabla \cdot 2 \mu \varepsilon(\boldsymbol{u}), \boldsymbol{v}_{h}\right)_{h} \\
& +\sum_{K}\left\langle 2 \mu \boldsymbol{n} \cdot \boldsymbol{\varepsilon}(\boldsymbol{u}),\left[\boldsymbol{v}_{h}\right]\right\rangle_{\partial K}-\sum_{K}\left\langle p,\left[\boldsymbol{n} \cdot \boldsymbol{v}_{h}\right]\right\rangle_{\partial K} \\
= & \left(\boldsymbol{f}, \boldsymbol{v}_{h}\right)+\sum_{K}\left\langle 2 \mu \boldsymbol{n} \cdot \boldsymbol{\varepsilon}(\boldsymbol{u}),\left[\boldsymbol{v}_{h}\right]\right\rangle_{\partial K}-\sum_{K}\left\langle p,\left[\boldsymbol{n} \cdot \boldsymbol{v}_{h}\right]\right\rangle_{\partial K}
\end{aligned}
$$

and thus the following Galerkin orthogonality relation holds:

$$
\begin{aligned}
a\left(\boldsymbol{u}-\boldsymbol{u}_{h}, \boldsymbol{v}_{h}\right)+j\left(\boldsymbol{u}-\boldsymbol{u}_{h}, \boldsymbol{v}_{h}\right)= & \sum_{K}\left\langle 2 \mu \boldsymbol{n} \cdot \boldsymbol{\varepsilon}(\boldsymbol{u}),\left[\boldsymbol{v}_{h}\right]\right\rangle_{\partial K} \\
& -\sum_{K}\left\langle p,\left[\boldsymbol{n} \cdot \boldsymbol{v}_{h}\right]\right\rangle_{\partial K} .
\end{aligned}
$$

We next define the norm

$$
\|\boldsymbol{u}\|:=\left\|\sigma^{1 / 2} \boldsymbol{u}\right\|^{2}+\left|\mu^{1 / 2} \boldsymbol{u}\right|_{h}^{2}+j(\boldsymbol{u}, \boldsymbol{u}),
$$

where

$$
\left|\boldsymbol{v}_{h}\right|_{h}^{2}:=\sum_{K}\left|\boldsymbol{v}_{h}\right|_{H^{1}(K)}^{2},
$$


$\left\|\boldsymbol{v}_{h}\right\|:=\left\|\boldsymbol{v}_{h}\right\|_{L_{2}(\Omega)}$, and, for use below,

$$
\left\|\boldsymbol{v}_{h}\right\|_{h}:=\left(\sum_{K}\left\|\boldsymbol{v}_{h}\right\|_{L_{2}(K)}^{2}\right)^{1 / 2} .
$$

By $r_{h}$ we denote the Crouzeix-Raviart interpolant,

$$
r_{h}:\left[H^{1}(\Omega)\right]^{d} \rightarrow V_{h}
$$

and note that if $\nabla \cdot \boldsymbol{u}=0$, then $r_{h}$ takes $\boldsymbol{u}$ into $W_{h}$ (cf. [4]).

We now wish to estimate the error measured in the triple norm, $\left\|\boldsymbol{u}-\boldsymbol{u}_{h}\right\| \mid$. Set

$$
\boldsymbol{e}_{h}:=r_{h} \boldsymbol{u}-\boldsymbol{u}_{h}
$$

and assume that $\boldsymbol{u} \in\left[H^{2}(\Omega)\right]^{d}$. By the triangle inequality

$$
\left\|\boldsymbol{u}-\boldsymbol{u}_{h}\right\|\left|\leq\left\|\boldsymbol{u}-r_{h} \boldsymbol{u}_{h}\right\|\right|+\left\|\boldsymbol{e}_{h}\right\| \mid .
$$

For $\left\|\boldsymbol{e}_{h}\right\| \mid$ we have the following result.

Lemma 4.1. There holds

$$
\left\|\boldsymbol{e}_{h} \mid\right\| \leq C\left(\left\|\boldsymbol{u}-r_{h} \boldsymbol{u}\right\| \mid+\mu^{1 / 2} h\|\boldsymbol{u}\|_{H^{2}(\Omega)}+h\|p\|_{H^{1}(\Omega)}\right)
$$

Proof. Define $\pi_{0}$ as the projection onto piecewise constants. By Korn's inequality for piecewise $H^{1}$ vector fields,

$$
\left|\mu^{1 / 2} \boldsymbol{v}_{h}\right|_{h}^{2} \leq C\left(\left\|\mu^{1 / 2} \varepsilon\left(\boldsymbol{v}_{h}\right)\right\|_{h}^{2}+j\left(\boldsymbol{v}_{h}, \boldsymbol{v}_{h}\right)\right),
$$

cf. Brenner [1], and the Galerkin orthogonality (4.1) we have

$$
\begin{aligned}
c\left\|\boldsymbol{e}_{h}\right\|^{2} \leq & \left\|\sigma^{1 / 2} \boldsymbol{e}_{h}\right\|^{2}+\left\|\mu^{1 / 2} \varepsilon\left(\boldsymbol{e}_{h}\right)\right\|_{h}^{2}+j\left(\boldsymbol{e}_{h}, \boldsymbol{e}_{h}\right) \\
= & a\left(\boldsymbol{e}_{h}, \boldsymbol{e}_{h}\right) \leq\left|a\left(r_{h} \boldsymbol{u}-\boldsymbol{u}_{h}, \boldsymbol{e}_{h}\right)\right|+j\left(r_{h} \boldsymbol{u}-\boldsymbol{u}_{h}, \boldsymbol{e}_{h}\right) \\
& +\left|\sum_{K}\left\langle 2 \mu \boldsymbol{n} \cdot \varepsilon(\boldsymbol{u}),\left[\boldsymbol{e}_{h}\right]\right\rangle_{\partial K}\right|+\left|\sum_{K}\left\langle p,\left[\boldsymbol{n} \cdot \boldsymbol{e}_{h}\right]\right\rangle_{\partial K}\right| \\
\leq & \left\|\boldsymbol{u}-r_{h} \boldsymbol{u}\right\|\left|\left\|\boldsymbol{e}_{h}\right\|\right|+\left|\sum_{K}\left\langle 2 \mu \boldsymbol{n} \cdot\left(\varepsilon(\boldsymbol{u})-\pi_{0} \varepsilon(\boldsymbol{u})\right),\left[\boldsymbol{e}_{h}\right]\right\rangle_{\partial K}\right| \\
& +\left|\sum_{K}\left\langle p-\pi_{0} p,\left[\boldsymbol{n} \cdot \boldsymbol{e}_{h}\right]\right\rangle_{\partial K}\right| \\
\leq & \left\|\boldsymbol{u}-r_{h} \boldsymbol{u}\right\|\left|\left\|\boldsymbol{e}_{h}\right\|\right| \\
& +\left(\sum_{K}\left\|2 \mu^{1 / 2} h^{1 / 2} \boldsymbol{n} \cdot\left(\varepsilon(\boldsymbol{u})-\pi_{0} \varepsilon(\boldsymbol{u})\right)\right\|_{\partial K}\right)^{1 / 2}\left\|\boldsymbol{e}_{h}\right\| \\
& +\left(\sum_{K}\left\|h^{1 / 2}\left(p-\pi_{0} p\right)\right\|_{\partial K}\right)^{1 / 2}\left\|\boldsymbol{e}_{h}\right\| \mid
\end{aligned}
$$

and, using the trace inequality

$$
\|v\|_{L_{2}(\partial K)}^{2} \leq C\left(h_{K}^{-1}\|v\|_{L_{2}(K)}^{2}+h_{K}\|v\|_{H^{1}(K)}^{2}\right), \quad \forall v \in H^{1}(K),
$$

we find

$$
\sum_{K}\left\|h^{1 / 2} \boldsymbol{n} \cdot\left(\varepsilon(\boldsymbol{u})-\pi_{0} \varepsilon(\boldsymbol{u})\right)\right\|_{\partial K}^{2} \leq \sum_{K} h_{K}^{2}\|\boldsymbol{u}\|_{2, K}^{2}
$$


and

$$
\sum_{K}\left\|h^{1 / 2}\left(p-\pi_{0} p\right)\right\|_{\partial K}^{2} \leq \sum_{K} h_{K}^{2}\|p\|_{1, K}^{2} .
$$

which ends the proof. $\square$

Lemma 4.2. For $\left\|\boldsymbol{u}-r_{h} \boldsymbol{u}\right\| \mid$ we have the following approximation result:

$$
\left\|\boldsymbol{u}-r_{h} \boldsymbol{u}\right\| \leq C\left(\sigma^{1 / 2} h^{2}+\mu^{1 / 2} h+\gamma^{1 / 2} \mu^{1 / 2} h\right)\|\boldsymbol{u}\|_{2}
$$

Proof. This follows from the trace inequality (4.4) and interpolation theory for $r_{h}$, see [4].

Combining Lemmas 4.1 and 4.2, we have the following a priori estimate.

THEOREM 4.3. Assuming that $\gamma_{\mu}$ and $\gamma_{0}$ are bounded from above and below, there holds

$$
\left\|\boldsymbol{u}-\boldsymbol{u}_{h}\right\| \leq C\left(\left(\sigma^{1 / 2} h^{2}+\mu^{1 / 2} h\right)\|\boldsymbol{u}\|_{H^{2}(\Omega)}+h\|p\|_{H^{1}(\Omega)}\right)
$$

For the pressure, we have the following estimate.

THEOREM 4.4. There holds

$$
\left\|p-p_{h}\right\|_{L_{2}(\Omega)} \leq C\left(\left(\sigma^{1 / 2} h^{2}+\mu^{1 / 2} h\right)\|\boldsymbol{u}\|_{H^{2}(\Omega)}+h\|p\|_{H^{1}(\Omega)}\right) .
$$

Proof. We split the error into

$$
\left\|p-p_{h}\right\| \leq\left\|p-\pi_{0} p\right\|_{L_{2}(\Omega)}+\left\|\pi_{0} p-p_{h}\right\|_{L_{2}(\Omega)},
$$

where $\pi_{0}$ is the $L_{2}$-projection of $p$ onto $Q_{h}$. For the first part we have the standard estimate

$$
\left\|p-\pi_{0} p\right\| \leq C h\|p\|_{H^{1}(\Omega)} .
$$

For the second part, we proceed as follows. By the surjectivity of the divergence operator (see [5]) there exists $\boldsymbol{v}_{p} \in\left[H_{0}^{1}(\Omega)\right]^{d}$ such that $\nabla \cdot \boldsymbol{v}_{p}=\pi_{0} p-p_{h}$ and $\left\|\boldsymbol{v}_{p}\right\|_{1, \Omega} \leq$ $C\left\|\pi_{0} p-p_{h}\right\|$. This gives, using the orthogonality of the $L_{2}$-projection,

$$
\begin{aligned}
\left\|\pi_{0} p-p_{h}\right\|^{2}= & \left(\pi_{0} p-p_{h}, \nabla \cdot \boldsymbol{v}_{p}\right)=\left(\pi_{0} p-p_{h}, \nabla \cdot r_{h} \boldsymbol{v}_{p}\right)_{h}=\left(p-p_{h}, \nabla \cdot r_{h} \boldsymbol{v}_{p}\right)_{h} \\
= & a_{h}\left(\boldsymbol{u}-\boldsymbol{u}_{h}, r_{h} \boldsymbol{v}_{p}\right)+j\left(\boldsymbol{u}-\boldsymbol{u}_{h}, r_{h} \boldsymbol{v}_{p}\right) \\
& +\sum_{K}\left\langle p,\left[\boldsymbol{n} \cdot r_{h} \boldsymbol{v}_{p}\right]\right\rangle_{\partial K}-\sum_{K}\left\langle 2 \mu \boldsymbol{n} \cdot \boldsymbol{\varepsilon}(\boldsymbol{u}),\left[r_{h} \boldsymbol{v}_{p}\right]\right\rangle_{\partial K}
\end{aligned}
$$

Using the trace inequality we find

$$
\begin{aligned}
a_{h}\left(\boldsymbol{u}-\boldsymbol{u}_{h}, r_{h} \boldsymbol{v}_{p}\right)+j\left(\boldsymbol{u}-\boldsymbol{u}_{h}, r_{h} \boldsymbol{v}_{p}\right) & \leq C\left\|\boldsymbol{u}-\boldsymbol{u}_{h}\right\|\left\|\boldsymbol{v}_{p}\right\|_{H^{1}(\Omega)} \\
& \leq C\left\|\boldsymbol{u}-\boldsymbol{u}_{h}\right\|\|\| \pi_{0} p-p_{h} \| \\
\sum_{K}\left\langle p,\left[\boldsymbol{n} \cdot r_{h} \boldsymbol{v}_{p}\right]\right\rangle_{\partial K} & =\sum_{K}\left\langle\left(p-\pi_{0} p\right),\left[\boldsymbol{n} \cdot r_{h} \boldsymbol{v}_{p}\right]\right\rangle_{\partial K} \\
& \leq \frac{1}{\gamma_{0}^{1 / 2}} \sum_{K} h_{K}^{1 / 2}\left\|p-\pi_{0} p\right\|_{\partial K}\left(\frac{\gamma_{0}}{h_{K}}\left\langle\left[\boldsymbol{n} \cdot r_{h} \boldsymbol{v}_{p}\right]\left[\boldsymbol{n} \cdot r_{h} \boldsymbol{v}_{p}\right]\right\rangle_{\partial K}\right)^{1 / 2} \\
& \leq C h\|p\|_{H^{1}(\Omega)}\left\|\pi_{0} p-p_{h}\right\|
\end{aligned}
$$


and

$$
\begin{aligned}
\sum_{K}\left\langle 2 \mu \boldsymbol{n} \cdot \varepsilon(\boldsymbol{u}),\left[r_{h} \boldsymbol{v}_{p}\right]\right\rangle_{\partial K} & \leq \sum_{K}\left\langle 2 \mu\left(\boldsymbol{n} \cdot \varepsilon(\boldsymbol{u})-\pi_{0} \boldsymbol{n} \cdot \varepsilon(\boldsymbol{u})\right),\left[r_{h} \boldsymbol{v}_{p}\right]\right\rangle_{\partial K} \\
& \leq C h\|\boldsymbol{u}\|_{H^{2}(\Omega)}\left\|\pi_{0} p-p_{h}\right\|,
\end{aligned}
$$

(where $\pi_{0} \boldsymbol{n} \cdot \varepsilon(\boldsymbol{u})$ is understood as the projection onto $\left[Q_{h}\right]^{d}$ ). Dividing both sides by $\left\|\pi_{0} p-p_{h}\right\|$ concludes the proof. $\square$

We end this section with an $L_{2}$-estimate for the velocities. We emphasize that this estimate can only be valid if $\mu$ is bounded from below since the necessary a priori regularity estimate does not hold otherwise.

Consider the dual continuous problem of seeking $\varphi$ and $r$ such that

$$
\begin{aligned}
\sigma \varphi-2 \mu \nabla \cdot \varepsilon(\varphi)+\nabla r & =\quad \text { in } \Omega, \\
\nabla \cdot \varphi & =0 \quad \text { in } \Omega
\end{aligned}
$$

where $\boldsymbol{e}:=\boldsymbol{u}-\boldsymbol{u}_{h}$, and assume that we have the regularity estimate

$$
\|\varphi\|_{H^{2}(\Omega)}+\|r\|_{H^{1}(\Omega)} \leq\|\boldsymbol{e}\| .
$$

Multiply the first line of (4.7) by $\boldsymbol{e}$ and integrate by parts to obtain

$$
\|\boldsymbol{e}\|^{2}=a_{h}(\boldsymbol{e}, \boldsymbol{\varphi})+j(\boldsymbol{e}, \boldsymbol{\varphi})-\sum_{K}\langle 2 \mu \boldsymbol{n} \cdot \boldsymbol{\varepsilon}(\boldsymbol{\varphi}),[\boldsymbol{e}]\rangle_{\partial K}+\sum_{K}\langle r,[\boldsymbol{n} \cdot \boldsymbol{e}]\rangle_{\partial K} .
$$

Using orthogonality and the zero mean value property of the Crouzeix-Raviart element, we obtain

$$
\begin{aligned}
\|\boldsymbol{e}\|^{2}= & a_{h}\left(\boldsymbol{e}, \boldsymbol{\varphi}-r_{h} \boldsymbol{\varphi}\right)+j\left(\boldsymbol{e}, \boldsymbol{\varphi}-r_{h} \boldsymbol{\varphi}\right)-\sum_{K}\left\langle 2 \mu\left(\boldsymbol{n} \cdot \boldsymbol{\varepsilon}(\boldsymbol{\varphi})-\pi_{0} \boldsymbol{n} \cdot \boldsymbol{\varepsilon}(\boldsymbol{\varphi})\right),[\boldsymbol{e}]\right\rangle_{\partial K} \\
& +\sum_{K}\left\langle r-\pi_{0} r,[\boldsymbol{n} \cdot \boldsymbol{e}]\right\rangle_{\partial K}-\sum_{K}\left\langle 2 \mu\left(\boldsymbol{n} \cdot \varepsilon(\boldsymbol{\varphi})-\pi_{0} \boldsymbol{n} \cdot \varepsilon(\boldsymbol{\varphi})\right),\left[\boldsymbol{\varphi}-r_{h} \boldsymbol{\varphi}\right]\right\rangle_{\partial K} \\
& +\sum_{K}\left\langle p-\pi_{0} p,\left[\boldsymbol{n} \cdot\left(\boldsymbol{\varphi}-r_{h} \boldsymbol{\varphi}\right)\right]\right\rangle_{\partial K} \\
\leq & \|\boldsymbol{e}\|\|\| \boldsymbol{\varphi}-r_{h} \boldsymbol{\varphi} \| \\
& +\left(\sum_{K} h_{K}\left\|2 \mu^{1 / 2}\left(\boldsymbol{n} \cdot \boldsymbol{\varepsilon}(\boldsymbol{\varphi})-\pi_{0} \boldsymbol{n} \cdot \boldsymbol{\varepsilon}(\boldsymbol{\varphi})\right)\right\|_{L_{2}(\partial K)}^{2}\right)^{1 / 2}\|\boldsymbol{e}\| \\
& +\left(\sum_{K} h_{K}\left\|r-\pi_{0} r\right\|_{L_{2}(\partial K)}\right)^{1 / 2}\|\boldsymbol{e}\| \\
& +\left(\sum_{K} h_{K}\left\|2 \mu^{1 / 2}\left(\boldsymbol{n} \cdot \boldsymbol{\varepsilon}(\boldsymbol{\varphi})-\pi_{0} \boldsymbol{n} \cdot \boldsymbol{\varepsilon}(\boldsymbol{\varphi})\right)\right\|_{L_{2}(\partial K)}^{2}\right)^{1 / 2}\left\|\boldsymbol{\varphi}-r_{h} \boldsymbol{\varphi}\right\| \\
& +\left(\sum_{K} h_{K}\left\|p-\pi_{0} p\right\|_{L_{2}(\partial K)}\right)^{1 / 2}\left\|\boldsymbol{\varphi}-r_{h} \boldsymbol{\varphi}\right\| .
\end{aligned}
$$

Using Lemma 4.2, the trace inequality and estimates for piecewise contant interpolation, we arrive at

$$
\|\boldsymbol{e}\|^{2} \leq C h\left(\|\boldsymbol{e}\|\|+\| \boldsymbol{u}\left\|_{H^{2}(\Omega)}+\right\| p \|_{H^{1}(\Omega)}\right)\left(\|\boldsymbol{\varphi}\|_{H^{2}(\Omega)}+\|r\|_{H^{1}(\Omega)}\right),
$$

and thus we have

THEOREM 4.5. Under the regularity assumption (4.8), the $L_{2}$-error in the velocities can be estimated as

$$
\|\boldsymbol{e}\| \leq C h^{2}\left(\|\boldsymbol{u}\|_{H^{2}(\Omega)}+\|p\|_{H^{1}(\Omega)}\right) .
$$




\section{Numerical results.}

5.1. Convergence study for Darcy flow. The first numerical example, taken from [8], is a study of convergence rates for Darcy flow. The domain under consideration is the unit square with a given exact pressure solution $p=-\sin \pi x+2 / \pi$ and velocity field $\boldsymbol{u}=\left(-\pi \sin 2 \pi y \sin ^{2} \pi x, \pi \sin 2 \pi x \sin ^{2} \pi y\right)$. We set $\gamma_{0}=1$. In Figure 5.1 , we show the convergence of the method in the $L_{2}-$ norm, which yields second order accuracy for the velocities and first order for the pressure. In Figure 5.2 we show the effect of removing the stabilizing normal jump. As pointed out in [8] there is in fact no convergence at all if $\gamma_{0}=0$.

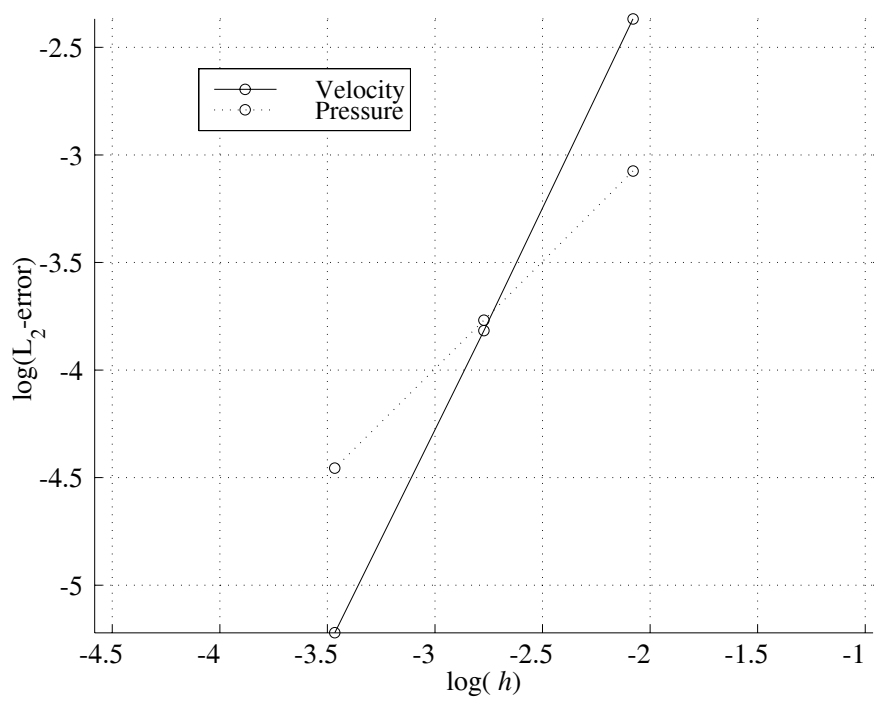

FIG. 5.1. Convergence in the Darcy case
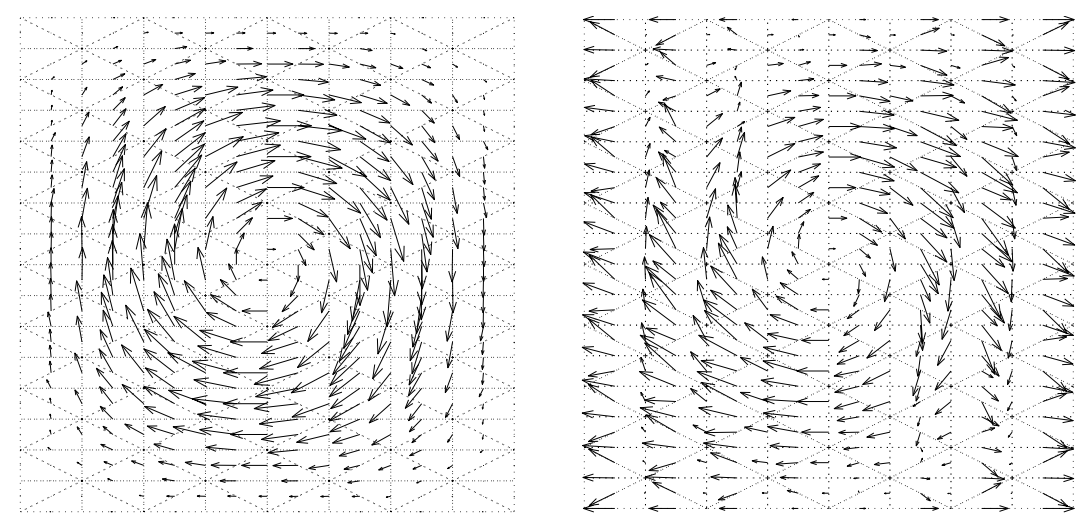

FIG. 5.2. Approximate solution of the velocities with and without normal jump stabilization

5.2. Convergence study for Stokes flow. Again, we consider the unit square with exact flow solution given by $u=\left(20 x y^{3}, 5 x^{4}-5 y^{4}\right)$ and $p=60 x^{2} y-20 y^{3}+C$. Choosing $\gamma_{\mu}=\gamma_{0}=1$ and imposing zero mean pressure $(C=-5)$, we obtain the optimal convergence shown in Figure 5.3. 


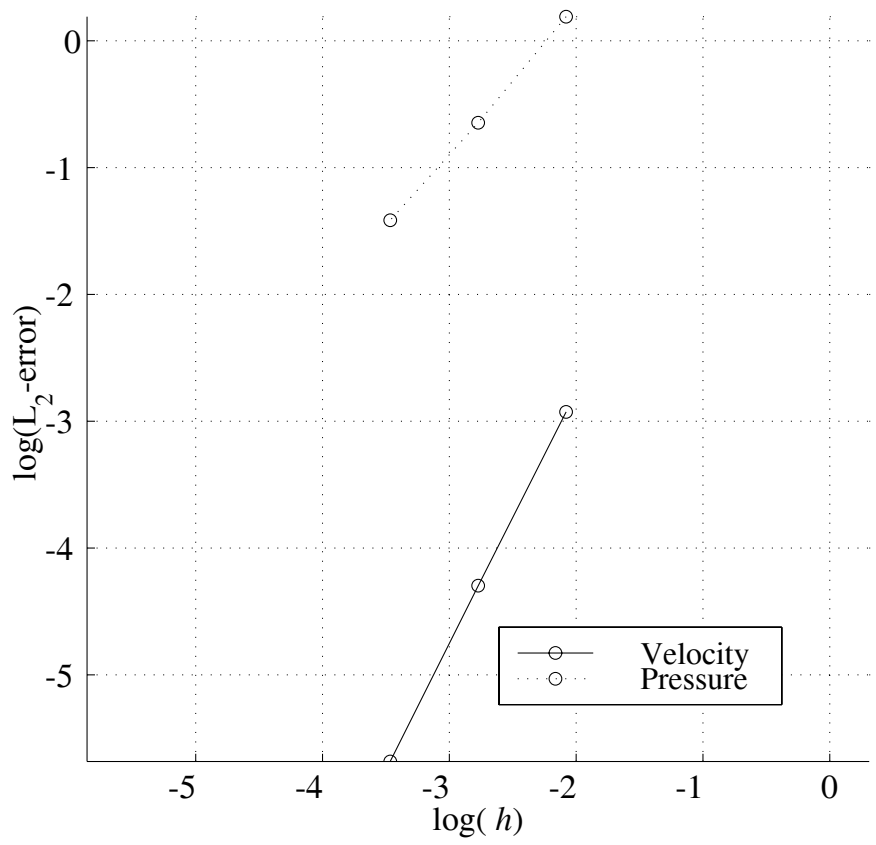

FIG. 5.3. $L_{2}$-norm convergence of the velocity and of the pressure for Stokes.

6. Acknowledgements. This paper was written while the second author was a guest of the Bernoulli center at the Ecole Polytechnique Fédérale de Lausanne, working within a program for the modeling of cardiovascular flow. The support of the Bernoulli center is gratefully acknowledged.

\section{REFERENCES}

[1] S. Brenner, Korn's inequalities for piecewise $H^{1}$ vector fields, Industrial Mathematics Research Report 2002:05, Department of Mathematics, University of South Carolina, 2002.

[2] F. Brezzi and M. Fortin, Mixed and Hybrid Finite Element Methods, Springer Verlag, New York, 1991.

[3] E. Burman and P. Hansbo, A unified stabilized method for Stokes' and Darcy's equations, Chalmers Finite Element Center Preprint 2002-15, Chalmers University of Technology, 2002.

[4] M. Crouzeix and P.-A. Raviart, Conforming and nonconforming finite element methods for solving the stationary Stokes equations. RAIRO Sér. Rouge 7 (1973) 33-75.

[5] V. Girault and P.-A. Raviart, Finite element methods for Navier-Stokes equations: Theory and algorithms, Springer-Verlag, Berlin, 1986.

[6] P. Hansbo and M.G. Larson, Discontinuous Galerkin methods for incompressible and nearly incompressible elasticity by Nitsche's method, Comput. Methods Appl. Mech. Engrg, 191, 1895-1908, 2002.

[7] P. Hansbo and M.G. Larson, Discontinuous Galerkin and the Crouzeix-Raviart element: Application to elasticity, M2AN Math. Model. Numer. Anal., 37, 63-72, 2003.

[8] K.A. Mardal, X.C. Tai, and R. Winther, A robust finite element method for Darcy-Stokes flow, SIAM J. Numer. Anal., 40, 1605-1631, 2002. 